\title{
Acolhimento em saúde: uma revisão sistemática em periódicos brasileiros
}

\author{
User embracement in health services: A systematic \\ review in Brazilian journals
}

\author{
Cátula PELISOLI ${ }^{1}$ \\ Airi Macias SACCO ${ }^{1}$ \\ Elen Teixeira BARBOSA ${ }^{2}$ \\ Cristiane de Oliveira PEREIRA ${ }^{2}$ \\ Alessandra Marques CECCONELLO ${ }^{2}$
}

\begin{abstract}
Resumo
Este estudo apresenta uma revisão sistemática sobre acolhimento em saúde em periódicos brasileiros. As bases de dados pesquisadas foram Bireme, MedLine, PubMed, SciELO e Science Direct. Utilizando como descritor único o termo "acolhimento", a pesquisa resultou na análise quantitativa e qualitativa de 40 artigos publicados entre 1999 e 2011. Os resultados indicaram que: os dois periódicos com maior número de publicações sobre o tema foram Cadernos de Saúde Pública e Ciência \& Saúde Coletiva (25,0 e 22,5\%, respectivamente); nos anos de 2004 a 2010, foram publicados sete artigos sobre o tema, e, em 2011, seis artigos foram encontrados. As áreas predominantes foram Saúde Pública/ Coletiva (57,6\%) e Enfermagem (39,4\%). A análise qualitativa da variável "objeto de estudo" identificou artigos cuja investigação era os pontos de vista de usuários, profissionais e de ambos. Já a variável "percepções dos profissionais" ressaltou aspectos positivos do acolhimento. São necessários novos estudos que qualifiquem o conhecimento a respeito dessa forma de atendimento à população tanto nos serviços de atenção básica quanto de atenção especializada.
\end{abstract}

Palavras-chave: Acolhimento; Saúde pública; Humanização da assistência; Sistema Único de Saúde.

\begin{abstract}
This study presents a systematic literature review on the subject "user embracement" in health in Brazilian journals. The databases analyzed were Bireme, PubMed, MedLine, SciELO, and Science Direct. The term "user embracement" was selected from the Health Sciences Descriptors and used as single descriptor. This resulted in 40 publications between 1999 and 2011, which were analyzed quantitatively and qualitatively. The results indicated that the two

$\boldsymbol{\nabla} \boldsymbol{\nabla} \boldsymbol{\nabla}$

1 Universidade Federal do Rio Grande do Sul, Instituto de Psicologia, Núcleo de Estudos e Pesquisas e Adolescência. R. Ramiro Barcelos, 2600/115, Santa Cecília, 90035-003, Porto Alegre, RS, Brasil. Correspondência para/Correspondence to: C. PELISOLI. E-mail: <catulapelisoli@yahoo.com.br>.

2 Centro de Atenção Psicossocial Casa Aberta. Osório, RS, Brasil.

Agradecimentos: À Secretaria da Saúde da Prefeitura de Osório, Rio Grande do Sul, em especial à Equipe de Saúde Mental e aos gestores que possibilitaram o desenvolvimento deste trabalho.
\end{abstract}


journals with more publications on the subject were Cadernos de Saúde Pública and Ciência \& Saúde Coletiva (25 and 22,5\%); seven papers were published in both 2004 and 2010 and six in 2011; the predominant areas were Public Health (57,6\%) and Nursing (39,4\%). The qualitative analysis of the "study object" variable identified the existence of articles investigating the views of users, professionals and both. Regarding the "professional' perceptions" variable, the majority of the articles highlighted positive aspects of user embracement. Further studies that qualify the knowledge about this form of service to the population, both in primary care services and specialized services, are necessary.

Keywords: User embracement; Public health; Humanization of assistance; Unified Health System.

A Política Nacional de Humanização (PNH) do Sistema Único de Saúde (SUS) define o termo "acolhimento" no campo da saúde como uma diretriz ética/estética/política e como uma ferramenta tecnológica de intervenção que procura qualificar a escuta, construir vínculos, garantir acesso com responsabilização e resolutividade nos serviços (Brasil, 2006). Trata-se de uma "tecnologia do encontro", com o objetivo de construir redes e processos de produção de saúde. O acolhimento propõe que as relações entre profissionais e usuários do SUS se transformem segundo uma base ética e de solidariedade, que sejam caracterizadas pelo reconhecimento do usuário como sujeito, como o participante ativo do processo de produção de saúde. Os objetivos do acolhimento são atender, ouvir pedidos, prestar atendimentos resolutivos responsáveis e estabelecer articulações entre os serviços da rede, encaminhando e orientando pacientes e familiares para a continuidade da assistência (Brasil, 2006).

O Humaniza-SUS é uma proposta de humanização que pretende alcançar as mais variadas ações e instâncias do SUS, que ocorrem nos diferentes níveis de atenção e de gestão (Brasil, 2009a). A humanização é compreendida como a valorização dos sujeitos implicados no processo de produção de saúde, cujos valores incluem autonomia, protagonismo, corresponsabilidade, vínculos solidários e participação coletiva (Brasil, 2009a). O acolhimento é um dispositivo de humanização que busca favorecer a construção de relações de confiança e de compromisso entre as equipes e os serviços, possibilitando avanços na aliança entre usuários, trabalhadores e gestores (Brasil, 2009b).

Como nova forma de gerir os processos de trabalho nos serviços de saúde, o acolhimento tem 226 sido implementado em diferentes contextos. No ambiente hospitalar, mulheres parturientes são acolhidas nos diferentes setores pelos quais transitam (Armellini \& Luz, 2003). Serviços especializados, como atendimento a pacientes com Acquired Immunodeficiency Syndrome (Aids), têm-se preocupado em conhecer características dos pacientes para a melhor estruturação dos serviços, assistência e otimização dos benefícios do cuidado (Braga, Cardoso, \& Segurado, 2007). Alguns autores mencionam a importância dos cuidados no acolhimento quanto ao gênero, pois a observância da especificidade das diferentes demandas geradas por homens e mulheres incide na qualidade da assistência prestada (Braga et al., 2007).

Com relação aos portadores de transtornos psíquicos na atenção básica, Brêda e Augusto (2001) concluíram que tanto profissionais de saúde quanto familiares parecem reproduzir a lógica manicomial, buscando a medicalização e a hospitalização. Nos serviços de atenção básica, é necessário investir na abordagem baseada na escuta, no acolhimento e no vínculo. Nesse contexto, a construção do vínculo depende tanto do paciente e de sua disponibilidade interna para o envolvimento quanto do profissional e de seus comportamentos de acolhida (Camelo, Angerami, Silva, \& Mishina, 2000). Em relação ao campo especializado da saúde mental, autores têm enfatizado o vínculo como um aspecto essencial da humanização (Jorge et al., 2011). Num Centro de Atenção Psicossocial (CAPS) do interior do Ceará, um estudo realizado com profissionais, familiares e usuários indicou o acolhimento como um dispositivo da relação de cuidado que contribui para a busca da resolução do problema (Jorge et al., 2011). Nesse trabalho, os autores enfocaram como as relações estabelecidas entre esses atores constroem vínculos que servem de base para os processos de comunicação e cuidado. A prática da tecnologia 
leve, segundo os autores desse estudo, deve ser fundamentada nas relações que se estabelecem no cuidado diário em saúde, no acolhimento, no diálogo, na corresponsabilização e na escuta ativa entre profissionais e usuários. Todos esses dispositivos são entendidos como ações comunicacionais, que incluem ouvir a população e dar respostas adequadas a ela. Para a área da saúde mental, o vínculo é fundamental e decisivo para os relacionamentos que aí se estabelecem. Visando a um cuidado integral, a saúde mental utiliza as relações de cuidado como ferramenta de sua prática. Nesse sentido, o CAPS busca potencializar a relação, visando à aproximação e a uma prática direcionada ao modo de vida do usuário e de sua família. Uma relevante observação dos autores é a de que o acolhimento não faz parte apenas do processo de triagem, mas atravessa todo o percurso do paciente dentro do sistema. O acolhimento é entendido como possibilidade de construir uma nova prática em saúde, e o funcionamento do tratamento do paciente é dependente do vínculo que é construído nessa relação (Jorge et al., 2011).

Não é só no CAPS, entretanto, que essa importância é revelada. Nos serviços de saúde, de modo geral, o relacionamento entre profissionais e a clientela é essencial para uma melhor assistência (Camelo et al., 2000). A relação de ajuda que se estabelece deve ser clara e tranquila; deve-se perceber o usuário como sujeito e agente das ações e construir um vínculo de acolhida e de responsabilização pelo cuidado integral em saúde individual e coletiva. O acolhimento é considerado uma tecnologia das relações ou uma tecnologia leve, com necessidade de respeito, resolutividade no atendimento e acesso às informações (Coelho \& Jorge, 2009). A produção de cuidado em saúde se dá na medida em que as relações se configuram entre usuários e trabalhadores e que ultrapassam o instrumental prescritivo (Merhy \& Franco, 2003). Nesse sentido, as tecnologias leves incluem o aspecto relacional que se dá entre as pessoas e que procuram a produção da saúde. Quando um trabalhador da saúde - além de prescrever exames, medicamentos e comportamento -, considera o sujeito como um todo, levando em conta sua origem social, relações familiares e sua subjetividade, ele está, nesse momento, usando as tecnologias leves, que permitem produzir o cuidado numa situação de acolhimento em saúde (Merhy \& Franco, 2003).

Nesse sentido, o acolhimento humanizado pressupõe a escuta sensível com intuito de aproximação e vinculação, observando o significado multidimensional da experiência vivenciada pelo usuário. Ao pesquisar a percepção de gestantes sobre o acolhimento, Armellini e Luz (2003) identificaram que o vínculo afetivo esperado pelas entrevistadas era caracterizado por uma relação amigável, com comunicação verbal e não verbal, com assuntos agradáveis, expressões de apoio e manifestações verbais acolhedoras, com toques, olhares e sorrisos. Esse processo pautado na disponibilidade, na aceitação, na empatia, no diálogo, na cordialidade, no respeito e na presteza representa tranquilidade e segurança para o paciente. A reedição do acolhimento demarca uma nova forma de relação entre profissionais da saúde e pacientes, sedimentada na subjetividade, na escuta das necessidades de quem busca ajuda e na corresponsabilização entre serviços de saúde, trabalhadores e usuários (Armellini \& Luz, 2003).

O conceito de humanização tem sido entendido como uma nova cultura de atendimento à saúde e uma forma de assistência que valoriza a qualidade do cuidado do ponto de vista técnico, incluindo o reconhecimento dos direitos dos pacientes, de sua subjetividade e cultura (Deslandes, 2004). Portanto, humanizar o atendimento significa se opor à violência da negação do outro e articular as tecnologias com o relacionamento, sendo o fator humano essencial na eficácia dos dispositivos organizacionais em saúde. Camelo et al. (2000) pressupõem que o acolhimento, que os autores chamam de "modelo de ajuda", inclua o atender, o responder, o personalizar, o orientar, o envolver-se, o explorar, o compreender e o agir. Quando eficaz, o encontro entre profissional e usuário é capaz de proporcionar o estabelecimento de uma relação de ajuda, na qual são mapeadas as demandas e as expectativas deste último.

Apesar de a proposta deste encontro humanizado parecer estar fundamentada em sólidas bases teóricas e metodológicas, algumas dificul- 
dades têm sido encontradas para que essa prática se torne realidade no SUS. A despeito de todos os esforços empreendidos até o momento, a escuta realizada no acolhimento continua sendo essencialmente clínica, inspirada no modelo biológico-médico-hegemônico, muito pontual e focada na queixa (Fracolli \& Zoboli, 2004). Habilidades técnicas são priorizadas em detrimento do acolhimento, e nem sempre as relações são personalizadas, pois cliente e auxiliar não se envolvem na relação (Camelo et al., 2000). Os profissionais dos diversos serviços ainda precisam aprender a reconhecer os problemas e as necessidades de saúde dos indivíduos e das famílias e traçar intervenções de maneira articulada entre os diferentes setores, instituindo um relacionamento com outros serviços da rede (Fracolli \& Zoboli, 2004).

Entretanto, resultados bastante positivos foram encontrados em uma experiência em acolhimento que tem influenciado muitas práticas nos serviços de saúde no Brasil: o caso de Betim (MG) (Franco, Bueno, \& Merhy, 1999). Nesse estudo, considerado um trabalho pioneiro, os autores observaram que, com (a) atendimento universal, (b) deslocamento do eixo central do médico para uma equipe multiprofissional, e (c) qualificação da relação trabalhador-usuário, ocorreu um incremento do rendimento profissional, maior oferta de serviços e aumento da acessibilidade. Outros aspectos positivos desse procedimento incluem o contato com pessoas, cujas demandas muitas vezes se diferenciam da própria área de formação do profissional; o comprometimento e a responsabilização da pessoa que realiza o acolhimento do paciente "acolhido"; e a possibilidade de uma discussão interdisciplinar do caso, o que aumenta, assim, as chances de uma resposta adequada aos problemas (Hennington, 2005). A perspectiva adotada pelo profissional pode ter influência direta sobre o encaminhamento adotado e, consequentemente, sobre a satisfação ou insatisfação das necessidades do sujeito atendido.

Com base na importância da implantação desse processo no SUS, este estudo tem como objetivo apreender o estado da arte do conhecimento e das experiências brasileiras dessa diretriz de humanização. A implantação da Política Nacional de Humanização deflagrou a necessidade de revisar a matéria a fim de entender como está sendo percebido o acolhimento como política e como prática no contexto da saúde brasileira. Para tanto, este trabalho apresenta, por meio de uma revisão sistemática da literatura, uma análise das publicações nacionais acerca do acolhimento.

\section{Método}

A revisão sistemática da literatura foi realizada nas bases de dados Bireme, MedLine, Pubmed, SciELO e Science Direct, indexadas pelo novo portal de periódicos da Coordenação de Aperfeiçoamento de Pessoal de Nível Superior (CAPES), do Governo Federal. Utilizou-se como descritor único o termo "acolhimento", selecionado por meio dos Descritores em Ciência da Saúde (DeCS), sendo este o único critério de inclusão do presente estudo. O processo de busca pelas publicações foi iniciado em 2010 e atualizado em 2012, incluindo todos os artigos publicados nessas bases de dados até o ano de 2011. Não foram considerados, portanto, artigos publicados a partir do ano de 2012.

Foram excluídos artigos que compreendiam o acolhimento institucional de crianças e adolescentes, que não é tema deste trabalho. Trata-se, portanto, de uma restrição em relação ao tema do estudo: o interesse dos autores é a investigação do tema acolhimento em saúde e não o antigamente denominado "abrigamento" (atualmente "acolhimento institucional") de crianças e adolescentes. A não observância desses trabalhos justifica-se pelo critério de exclusão de todos e quaisquer artigos que não se referissem ao acolhimento no contexto dos serviços de saúde.

Inicialmente, foram encontrados 81 artigos com a utilização desse único descritor. Após uma primeira análise, verificou-se que alguns deles não continham o termo nem no título, nem no resumo, e outros não se referiam ao acolhimento em saúde. Dessa forma, foram consideradas 40 publicações (aquelas que foram citadas no texto foram referenciadas e indicadas por meio de um asterisco na 
lista de referências) que tiveram suas variáveis analisadas quantitativa e qualitativamente. Dentre as variáveis analisadas quantitativamente, através de estatística descritiva, estão: (1) ano de publicação; (2) região brasileira de origem do estudo; (3) método utilizado na pesquisa; (4) área da saúde responsável pela publicação; e (5) periódico em que o trabalho foi publicado. As variáveis analisadas qualitativamente foram (a) objetivo; e (b) principais resultados. Ambas foram submetidas à análise de conteúdo (Bardin, 2009).

\section{Resultados e Discussão}

Foram encontrados trabalhos publicados a partir do ano de 1999, sendo o primeiro artigo encontrado o referente à experiência de Betim (MG), retratada aqui neste trabalho e conhecida no país como tendo um caráter pioneiro. De 1999 até 2002, cinco artigos sobre acolhimento foram publicados nas bases de dados pesquisadas (Figura 1). Em 2003 e 2004, houve uma intensificação na produção de artigos que abordaram esse tema, o que talvez possa ser explicado pela crescente valorização do acolhimento como uma ferramenta da atenção e da gestão que contribui com a regulação do acesso, com a promoção de vínculo com o usuário e com a oferta de cuidado mais adequado, além de funcionar como dispositivo analisador dos serviços de saúde (Brasil, 2006). No ano de 2004, a PNH certamente impulsionou as publicações na área, uma vez que se constitui como um marco para o acolhimento em saúde. De 2005 a 2009, o número

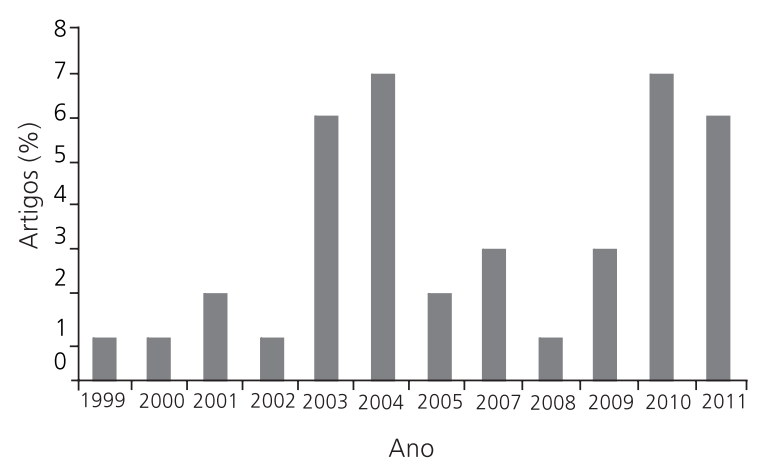

Figura 1. Distribuição, até 2011, da frequência absoluta dos artigos por ano de publicação. diminuiu em relação aos anos anteriores, mas, com exceção de 2006, ao menos um artigo foi publicado por ano. Em 2010 e 2011, houve um novo pico de publicações, com ênfase em análises críticas sobre a prática do acolhimento em unidades de saúde. Mais uma vez, as publicações do Ministério da Saúde podem ter impulsionado a escrita, a reflexão e as publicações por parte dos pesquisadores e trabalhadores da área (Brasil, 2009a, 2009b).

A produção acadêmica sobre o acolhimento tem-se concentrado nas regiões Sudeste $(42,4 \%)$ e Sul $(36,4 \%)$, seguidas pela Nordeste $(15,1 \%)$ e pela Centro-Oeste $(6,1 \%)$. Não foram encontrados registros de estudos da região Norte. Em relação ao tipo de serviço estudado junto à rede de atenção, 55,5\% dos estudos foram realizados na atenção básica, e 45,5\% englobaram o nível ambulatorial e hospitalar.

No que se refere ao método utilizado, há uma predominância de estudos qualitativos (97,5\%; $\mathrm{n}=39$ ). Apesar de a maior parte deles não descrever os procedimentos adotados, foi possível identificar relatos de experiência, análise documental, estudos de caso e pesquisa bibliográfica. Dentre os 40 artigos pesquisados, três apresentaram abordagem quali-quantitativa, e apenas um era exclusivamente quantitativo. $\mathrm{O}$ estudo quantitativo encontrado foi o de Braga et al. (2007), que trata do acolhimento de pessoas com Aids. Nesse estudo, foram investigados 1072 prontuários de pacientes para avaliar o acesso ao serviço de referência, com o foco em diferenças de sexo. Os autores encontraram que, em comparação aos homens, as mulheres buscam atendimento em estágios clínicos menos avançados da doença.

O fato de termos apenas um estudo quantitativo pode ser explicado pela observação de que o acolhimento, por ser uma tecnologia leve, que faz parte do campo relacional entre o técnico e o usuário, considera questões de ordem subjetiva e que, portanto, direciona os pesquisadores para a realização de estudos qualitativos na tentativa de compreender ampla e profundamente o processo. Assim, os pesquisadores parecem considerar que a abordagem qualitativa é a mais adequada para dar 
conta desse objeto de estudo e para apreender melhor essa realidade. Além disso, como boa parte das unidades de saúde ainda está tentando implantar um modelo de acolhimento realmente efetivo, estudos qualitativos, principalmente relatos de experiência, podem exercer um importante papel no compartilhamento de experiências bem-sucedidas e malsucedidas, que sirvam de exemplo para outros profissionais. Entretanto, ressalta-se que as pesquisas quantitativas e a elaboração de instrumentos para uso em larga escala também podem apresentar contribuições importantes para a área.

Com relação às áreas da saúde que se ocupam da construção do saber em relação à prática do acolhimento, a enfermagem é a que mais se destaca (37,5\%, n=15) (Figura 2). A área da Psicologia/Saúde Mental foi responsável por quatro publicações, e a Odontologia, por três. Quase metade das produções refere-se ao acolhimento como prática da saúde pública/saúde coletiva, ou seja, como temática de conhecimento de campo. Essa realidade está refletida também nos periódicos de divulgação dos artigos, pois mais da metade dos estudos (57,5\%, n=23) foi publicada em periódicos de saúde pública/coletiva ou ainda periódicos interdisciplinares. As revistas especializadas da área da enfermagem apresentaram um número considerável de estudos em relação a outras áreas da saúde. Historicamente, o atendimento à saúde se baseia em um modelo assistencial centrado no papel do médico, no qual a enfermagem, além de não desempenhar todo seu potencial, tem uma reduzida capacidade de intervenção. Contudo, após o advento do acolhimento na saúde pública brasileira, a enfermagem teve seu papel consideravelmente valorizado (Franco et al., 1999), e essa trajetória é uma das possíveis razões pelas quais essa área tem-se destacado no que diz respeito às publicações nacionais.

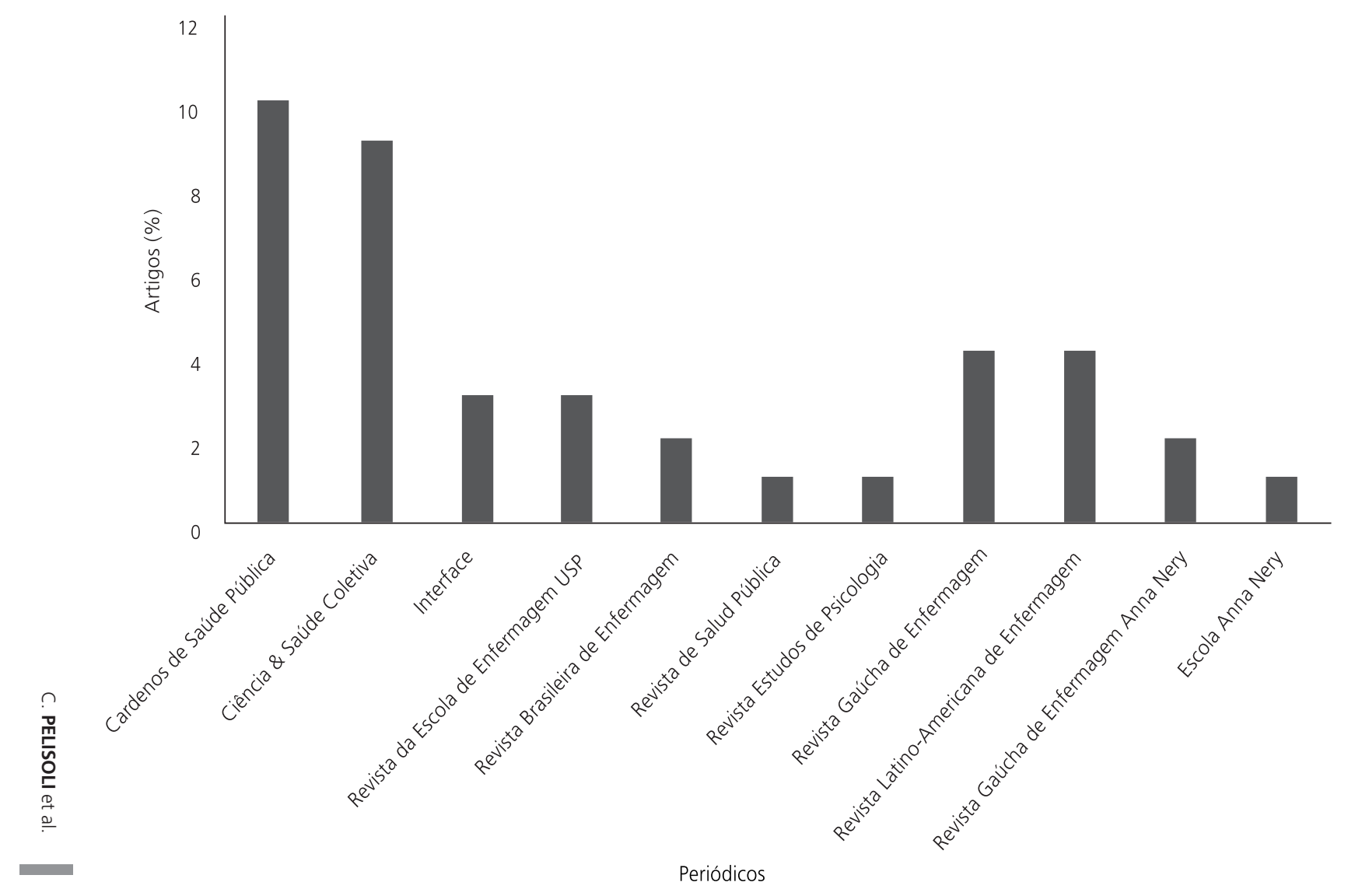

230 Figura 2. Distribuição da frequência absoluta dos artigos por periódico até 2011. 
Com relação à análise qualitativa, foram abordadas duas variáveis: "objeto de estudo" e "percepções de profissionais". A análise dos objetos de estudo dos artigos encontrados permitiu verificar o estado da arte do conhecimento sobre acolhimento em saúde no Brasil (Quadro 1). Foram identificados três eixos temáticos principais: pessoas, documentos e experiências. Com relação ao eixo pessoas, alguns artigos coletaram dados exclusivamente de pacientes, outros exclusivamente de profissionais, e alguns poucos avaliaram percepções tanto de uns quanto de outros. O segundo eixo documentos -, diz respeito aos estudos que incluíram análises documentais em suas avaliações, e o terceiro - experiências -, faz referência àqueles que relataram experiências de acolhimento.

Nos artigos que investigaram o acolhimento a partir da perspectiva do paciente/usuário, os trabalhos encontrados abordaram parturientes, famílias com filhos excepcionais, acompanhantes de pacientes e usuários de uma unidade básica de saúde. Somente em 2011, foi encontrado um artigo que abordava as percepções de usuários de um
Centro de Atenção Psicossocial sobre o acolhimento recebido. Outro artigo investigou portadores de transtornos psíquicos em uma unidade básica de saúde.

Dentre os profissionais que atuam na área da saúde, os da enfermagem têm-se apropriado muito mais da questão do acolhimento do que outros, como médicos, psicólogos, dentistas ou assistentes sociais. Estudos com profissionais de diferentes áreas, caracterizando uma equipe interdisciplinar, também são raros. Tendo em vista que o acolhimento é um procedimento que pode e deve ser exercido por diferentes profissionais da área da saúde e considerando a necessidade de uma escuta dinâmica e generalizada, portanto não especializada, para as diferentes demandas do paciente, é necessário que o campo seja mais explorado também por outras áreas do conhecimento. Na categoria documentos, uma importante observação é a de que, mais uma vez, a área da enfermagem encontra espaço destacado, situando o acolhimento como ponto importante dentro do seu histórico.

\section{Quadro 1}

Análise da variável "objeto de estudo"

\begin{tabular}{lll}
\hline Eixos temáticos & Categorias & Subcategorias \\
\hline Pessoas & Usuários & Parturientes \\
& Filhos excepcionais \\
& Acompanhantes de pacientes \\
& Usuários de Unidade de Saúde \\
& Grupos interdionais & Área da enfermagem \\
& Saúde da família \\
& Plantão psicológico \\
& Cirurgiões-dentistas \\
& Portadores de transtornos psíquicos/saúde mental \\
& Atenção básica \\
& Tecnologia informacional e qualidade do atendimento \\
& Saúde bucal \\
Análise documental & Prontuários \\
& Documentos do Ministério da Saúde \\
& Histórico da enfermagem \\
& Artigos científicos \\
& Registros institucionais \\
& Diários de campo \\
& Betim, Minas Gerais \\
& Pipas, Rio Grande do Sul \\
& Unidade Básica de Saúde \\
\hline
\end{tabular}


Outra relevante lacuna identificada a partir dos resultados desta revisão diz respeito à escassez de relatos de experiências publicados: foram encontrados apenas três, sendo um deles o caso exponencial de Betim (MG) (Franco et al., 1999). A importância dos serviços divulgarem suas formas de proceder reside na possibilidade de multiplicação de experiências bem-sucedidas. Dessa forma, é interessante que as unidades divulguem suas tecnologias de atendimento tanto no meio acadêmico quanto no meio técnico, que é aquele com potencial para beneficiar diretamente os usuários.

A segunda categoria analisada qualitativamente teve como intuito compreender como o acolhimento é percebido pelos profissionais que trabalham na área da saúde (Quadro 2). De maneira geral, os estudos descreveram o acolhimento como uma ferramenta necessária tanto nas unidades bási- cas de saúde e nas estratégias de saúde da família quanto nos hospitais, especialmente no momento da parturição e no nascimento de crianças com deficiência.

Alguns estudos descreveram o acolhimento como um campo interdisciplinar, que pode ser realizado por diversos profissionais da saúde, como médicos, enfermeiros, psicólogos e dentistas. A maior parte deles, no entanto, relaciona o acolhimento com um núcleo de conhecimento específico: a enfermagem. Corroborando os dados provenientes da análise quantitativa, esses resultados apresentam a dificuldade do envolvimento multidisciplinar. Podem indicar ainda que, em alguns locais, o termo acolhimento esteja sendo considerado análogo à triagem, atividade que, usualmente, é de responsabilidade de profissionais da enfermagem. Outra possibilidade é a de ainda prevalecer o

Quadro 2

Análise da categoria "percepções de profissionais"

\begin{tabular}{|c|c|c|}
\hline Eixos temáticos & Categorias & Subcategorias \\
\hline \multirow{5}{*}{$\begin{array}{l}\text { Acolhimento como fer- } \\
\text { ramenta necessária }\end{array}$} & \multirow{3}{*}{ Nas UBS e ESF } & Na saúde mental \\
\hline & & Na saúde bucal \\
\hline & & Com pacientes HIV e familiares \\
\hline & \multirow[t]{2}{*}{ Nos hospitais } & No momento da parturição \\
\hline & & No nascimento de crianças com deficiência \\
\hline \multirow{2}{*}{$\begin{array}{l}\text { Acolhimento e campo de co- } \\
\text { nhecimento }\end{array}$} & Como campo interdisciplinar & Áreas da saúde \\
\hline & $\begin{array}{l}\text { Como núcleo de conhecimento } \\
\text { específico }\end{array}$ & Ligado à enfermagem \\
\hline \multirow{14}{*}{$\begin{array}{l}\text { Acolhimento como disposi- } \\
\text { tivo analisador }\end{array}$} & \multirow[t]{10}{*}{ Aspectos negativos } & Intervenção pontual, pouco resolutiva \\
\hline & & Falta de vínculo \\
\hline & & $\begin{array}{l}\text { Lacunas referentes à estruturação do serviço (rotinas, abertura, informa- } \\
\text { tização) e necessidade de reorganização do serviço e da rede }\end{array}$ \\
\hline & & Falta de implicação do profissional na resolução dos problemas \\
\hline & & Presença de sofrimento e adoecimento por parte do trabalhador \\
\hline & & Longa espera por parte do usuário \\
\hline & & Etapa de organização da demanda \\
\hline & & Estratégia para o gerenciamento da qualidade do serviço \\
\hline & & Estruturação do serviço que permite aprimorar a assistência \\
\hline & & Maior acessibilidade do usuário \\
\hline & \multirow{4}{*}{ Aspectos positivos } & Humanização do atendimento \\
\hline & & $\begin{array}{l}\text { Postura na relação com o usuário, de sensibilidade, compromisso, solidarie- } \\
\text { dade, responsabilidade, respeito e cidadania }\end{array}$ \\
\hline & & Proporciona a melhora do paciente e mudanças positivas \\
\hline & & Construção de espaço de diálogo entre trabalhador, familiar e usuário \\
\hline
\end{tabular}

232 Note: UBS: Unidade Básica de Saúde; ESF: Estratégia de Saúde da Família; HIV: Human Immunodeficiency Vírus. 
interesse por tecnologias duras por parte dos demais profissionais, já que essas são, historicamente, mais valorizadas no campo da saúde. Nesse caso, estaria a enfermagem muito mais envolvida com as situações de acolhimento do que outras disciplinas da saúde. Idealmente, contudo, enquanto a triagem é apenas uma parte do processo de atendimento ao usuário, o acolhimento deve perpassar todas as etapas (Pereira et al., 2010) e estar relacionado à postura ética do profissional, independentemente de sua formação acadêmica (Ribeiro, Rocha, \& Ramos-Jorge, 2010).

Ainda com relação à análise qualitativa, foram identificados aspectos negativos e positivos associados ao acolhimento. Entre os aspectos negativos, o acolhimento foi descrito como uma intervenção pontual, pouco resolutiva e não construtora de vínculo (Fracolli \& Zoboli, 2004), e também foi relacionado com uma falta de implicação do profissional na resolução dos problemas dos pacientes (Barros \& Sá, 2010; Schimith \& Lima, 2004) e com uma falta de estruturação do serviço e de aspectos como rotinas, abertura e informatização (Marciel-Lima, 2004). Além disso, alguns autores identificaram uma redução do acolhimento a procedimentos de recepção, triagem e encaminhamento (Brehmer \& Verdi, 2010).

Dentre os pontos positivos, foi destacada a estruturação do serviço proporcionada pelo acolhimento, a qual permite o aprimoramento da assistência, a maior acessibilidade do usuário e a humanização do atendimento (Rossi \& Lima, 2005; Takemoto \& Silva, 2007). O acolhimento também foi relacionado a uma postura de sensibilidade, compromisso, solidariedade, responsabilidade, respeito e cidadania na relação com o usuário, o que contribui para a melhora do paciente (Silveira \& Carvalho, 2002; Trevizan, Mendes, Lourenço, \& Melo, 2003).

Nota-se que, entre os pontos positivos e negativos, existem contradições, como os aspectos que se referem, por exemplo, à relação do profissional com o usuário. Enquanto um artigo afirma que há falta de implicação do primeiro para com o último, outros mostram que a postura de cuidado e de comprometimento caracteriza a particularidade desse dispositivo. Tendo em vista os aspectos teóricos delineados no decorrer deste trabalho, pode-se concluir que não há acolhimento propriamente dito, como é entendido pelo Ministério da Saúde (Brasil, 2006; 2009a; 2009b), se não houver uma relação de proximidade com o usuário. Cabe aos pesquisadores e aos trabalhadores da área compartilhar as informações que contribuam para o conhecimento da realidade da prática do acolhimento no Brasil, e mostrar as dificuldades e as virtudes de sua implementação nos mais diferentes serviços de saúde.

O presente estudo realizou uma revisão sistemática da literatura nacional. Como limitações desse método de investigação destaca-se que não são coletados diretamente dados empíricos dos serviços, dos profissionais ou dos usuários do Sistema Único de Saúde, sendo, portanto, utilizados dados já publicados. Uma das limitações deste estudo, especificamente, foi a não avaliação da qualidade metodológica dos artigos encontrados. Outra limitação é a utilização de poucos critérios de inclusão dos artigos, uma vez que o objetivo foi apreender o estado da arte desse tema no Brasil.

Este estudo demonstrou que, apesar de a produção científica na área ainda ser relativamente escassa, há uma preocupação crescente com o assunto, especialmente nos últimos três anos. Os estudos reconhecem a importância do acolhimento para a humanização do atendimento e das relações no sistema de saúde. De maneira geral, no entanto, alguns autores concordam que há um distanciamento entre o que é proposto na teoria e o que é colocado em prática (Brehmer \& Verdi, 2010; Pereira et al., 2010; Ribeiro et al., 2010).

Para que essa lacuna diminua, os serviços precisam implementar um espaço físico acolhedor, realizar treinamento de trabalhadores a fim de humanizar a atenção e promover atividades transversais de acolhimento (Coelho \& Jorge, 2009). Essa prática deveria permear todo o percurso do usuário no sistema de saúde, mas, muitas vezes, fica restrita ao primeiro atendimento, realizado por profissionais de enfermagem. Essa realidade ficou evidente na análise das áreas e periódicos responsáveis pelas publicações sobre o tema. Ainda são poucos os es- 
tudos sobre acolhimento conduzidos por psicólogos, médicos, odontólogos, assistentes sociais ou mesmo por equipes interdisciplinares, como seria o ideal. Um exemplo disso é a escassez de pesquisas que envolvem usuários de Centros de Atenção Psicossocial. Nesse sentido, é imprescindível investir no preparo dos profissionais que atuam nos serviços de saúde, principalmente no que diz respeito ao relacionamento interpessoal e à comunicação terapêutica, a fim de favorecer sua receptividade ao trabalho em equipe e a expressão das demandas subjetivas de usuários, familiares e demais profissionais, a partir de uma escuta ampliada (Brêda \& Augusto, 2001; Fracolli \& Zoboli, 2004).

Para que o acolhimento torne-se uma realidade junto aos serviços brasileiros de saúde, é necessário incentivar a divulgação de casos bem-sucedidos. A publicação de relatos de experiência é essencial para que os profissionais que estão na ponta do processo tenham em quem se espelhar. Da mesma forma, também são importantes estudos de avaliação de impacto e de processo desta que é considerada uma tecnologia leve, pautada em relações de cuidado e atenção (Rossi \& Lima, 2005).

Em termos teóricos, o acolhimento representa um avanço substancial em direção à humanização do atendimento em saúde no Brasil. Se, contudo, ainda falta muito para que esse potencial seja desenvolvido em sua plenitude, cabe ao meio acadêmico dar sua contribuição para esse processo, promovendo estudos sobre o tema e difundindo seus resultados para a sociedade.

\section{Referências}

Armellini, C. J., \& Luz, A. M. H. (2003). Acolhimento: a percepção das mulheres na trajetória da parturição. Revista Gaúcha de Enfermagem, 24(3), 305-315.

Bardin, L. (2009). Análise de conteúdo. Lisboa: Edições 70.

Barros, D. M., \& Sá, M. C. (2010). O processo de trabalho em saúde e a produção do cuidado em uma unidade de saúde da família: limites ao acolhimento e reflexos no serviço de emergência. Ciência e Saúde Coletiva, 15(5), 2473-2482.

Braga, P. E., Cardoso, M. R. A., \& Segurado, A. C. (2007). Diferenças de gênero ao acolhimento de pessoas viven- do com HIV em serviço universitário de referência de São Paulo, Brasil. Cadernos de Saúde Pública, 23(11), 2653-2662.

Brasil. Ministério da Saúde. (2006). Acolhimento nas práticas de produção de saúde. Brasília: Ministério da Saúde.

Brasil. Ministério da Saúde. Secretaria de Atenção à Saúde. (2009a). O humaniza SUS na atenção básica. Brasília: Ministério da Saúde. Recuperado em janeiro 28, 2012, de http://bvsms.saude.gov.br/bvs/publicacoes/ humaniza_sus_atencao_basica.pdf

Brasil. Ministério da Saúde. Secretaria de Atenção à Saúde. (2009b). Acolhimento e classificação de risco nos serviços de urgência. Brasília: Ministério da Saúde. Recuperado em janeiro 28, 2012, de http://bvsms. saude.gov.br/bvs/publicacoes/acolhimento_ classificaao_risco_servico_urgencia.pdf

Brêda, M. Z., \& Augusto, L. G. S. (2001). O cuidado ao portador de transtorno psíquico na atenção básica de saúde. Ciência \& Saúde Coletiva, 6(2), 471-480.

Brehmer, L. C. F., \& Verdi, M. (2010). Acolhimento na atenção básica: reflexões éticas sobre a atenção à saúde dos usuários. Ciência \& Saúde Coletiva, 15(Supl. 3), 3569-3578.

Camelo, S. H. H., Angerami, E. L. S., Silva, E. M., \& Mishima, S. M. (2000). Acolhimento à clientela: estudo em unidades básicas de saúde no município de Ribeirão Preto. Revista Latino-Americana de Enfermagem, 8(4), 30- 37.

Coelho, M. O., \& Jorge, M. S. B. (2009). Tecnologia das relações como dispositivo do atendimento humanizado na atenção básica à saúde na perspectiva do acesso, do acolhimento e do vínculo. Ciência \& Saúde Coletiva, 14(Supl. 1), 1523-1531.

Deslandes, S. F. (2004). Análise do discurso oficial sobre a humanização da assistência hospitalar. Ciência \& Saúde Coletiva, 9(1), 7-14.

Fracolli, L. A., \& Zoboli, E. P. (2004). Descrição e análise do acolhimento: uma contribuição para o programa de saúde da família. Revista da Escola de Enfermagem da USP, 38(2),143-151.

Franco, T. B., Bueno, W. S., \& Merhy, E. E. (1999). O acolhimento e os processos de trabalho em saúde: o caso de Betim, Minas Gerais, Brasil. Cadernos de Saúde Pública, 15(2), 345-353.

Hennington, E. A. (2005). Acolhimento como prática interdisciplinar num programa de extensão universitária. Cadernos de Saúde Pública, 21(1), 256-265.

Jorge, M. S. B., Pinto, D. M., Quinderé, P. H. D., Pinto, A. G. A., Sousa, F. S. P., \& Cavalcante, C. M. (2011). Promoção da saúde mental: tecnologias do cuidado: vínculo, acolhimento, co-responsabilização e autonomia. Ciência e Saúde Coletiva, 16(7), 3051-3060. 
Marciel-Lima, S. M. (2004). Acolhimento solidário ou atropelamento? A qualidade na relação profissional de saúde e paciente face à tecnologia informacional. Caderno de Saúde Pública, 20(2), 502-511.

Merhy, E. E., \& Franco, T. B. (2003). Por uma composição técnica do trabalho centrada nas tecnologias leves e no campo relacional. Saúde em Debate, 27(65), 345-353.

Pereira, A. D., Freitas, H. M. B., Ferreira, C. L. L., Marchiori, M. R. C. T., Souza, M. H. T., \& Backes, D. S. (2010). Atentando para as singularidades humanas na atenção à saúde por meio do diálogo e acolhimento. Revista Gaúcha de Enfermagem, 31(1), 55-61.

Ribeiro, L. C. C., Rocha, R. L., \& Ramos-Jorge, M. L. (2010). Acolhimento às crianças na atenção primária à saúde: um estudo sobre a postura dos profissionais das equipes de saúde da família. Cadernos de Saúde Pública, 26(12), 2316-2322.

Rossi, F. R., \& Lima, M. L. (2005). Acolhimento: tecnologia leve nos processos gerenciais do enfermeiro. Revista Brasileira de Enfermagem, 58(3), 305-310.
Schimith, M. D., \& Lima, M. S. (2004). Acolhimento e vínculo em uma equipe do programa saúde da família. Cadernos de Saúde Pública, 20(6), 1487-1494.

Silveira, E. A. A., \& Carvalho, A. M. P. (2002). Familiares de clientes acometidos pelo HIV/AIDS e o atendimento prestado em uma unidade ambulatorial. Revista Latino-Americana de Enfermagem, 10(6), 813-818.

Takemoto, M. L. S., \& Silva, E. M. (2007). Acolhimento e transformações no processo de trabalho de enfermagem em unidades básicas de saúde de Campinas, São Paulo, Brasil. Cadernos de Saúde Pública, 23(2), 331-340.

Trevizan, M. A., Mendes, I. A. C., Lourenço, M. R., \& Melo, M. C. (2003). Al encuentro de la competencia del cuidado según Boff: una nueva perspectiva de conducta ética de la enfermera gerente. Revista Latino-Americana de Enfermagem, 11(5), 652-657.

Recebido em: 2/2/2012

Versão final em: 22/5/2012

Aprovado em: 12/6/2012 
